\title{
Expression and clinical significance of aquaglyceroporins in human hepatocellular carcinoma
}

\author{
XIAO-FENG CHEN, CHUAN-FEI LI, LIN LÜ and ZHE-CHUAN MEI \\ Department of Gastroenterology, The Second Affiliated Hospital, Chongqing Medical University, \\ Chongqing 400010, P.R. China
}

Received April 22, 2015; Accepted April 13, 2016

DOI: $10.3892 / \mathrm{mmr} .2016 .5184$

\begin{abstract}
Aquaglyceroporins (AQPs) are a subset of the aquaporin family, and are permeable to water and glycerol. The aim of the present study was to determine the expression and clinical significance of three AQPs, AQP3, 7 and 9 in hepatocellular carcinoma (HCC). Fresh HCC and adjacent non-tumorous liver tissues were collected from 68 patients diagnosed with HCC. The expression levels of AQP3, 7 and 9 were detected by reverse transcription-quantitative polymerase chain reaction, western blotting and immunohistochemical analysis. The association between the expression of AQPs and clinicopathological parameters of HCC were investigated. Compared with non-tumorous liver tissue, HCC tissues exhibited a significant $(\mathrm{P}<0.05)$ increase in the expression of AQP3 and a concomitant reduction in the expression levels of AQP7 and AQP9, at both the mRNA and protein levels. Immunohistochemistry revealed that AQP9 was dominantly localized on the plasma membrane of hepatocytes, while AQP3 and AQP7 exhibited a predominantly cytoplasmic and nuclear distribution. High expression of AQP3 was significantly $(\mathrm{P}<0.05)$ associated with low expression levels of AQP7 and AQP9. High expression of AQP3 was correlated with tumor grade $(\mathrm{P}=0.017)$, tumor stage $(\mathrm{P}=0.010)$ and lymphatic metastasis $(\mathrm{P}=0.031)$. Low expression of AQP7 was correlated with tumor grade $(\mathrm{P}=0.043)$. AQP3 was upregulated, and AQP7 and AQP9 were downregulated in HCC. A high expression of AQP3 and low expression of AQP7 was significantly associated with the aggressive features of HCC.
\end{abstract}

Correspondence to: Dr Zhe-Chuan Mei, Department of Gastroenterology, The Second Affiliated Hospital, Chongqing Medical University, 76 Linjiang Road, Chongqing 400010, P.R. China

E-mail: meizhechuan@21cn.com

Key words: aquaglyceroporins, clinical significance, expression regulation, hepatocellular carcinoma

\section{Introduction}

Aquaporins are integral membrane channel proteins that facilitate transcellular water movements (1). To date, 13 AQPs (AQP0-AQP12) have been cloned in mammals. $\mathrm{AQP} 3,7,9$ and 10 constitute the aquaglyceroporin (AQP) subfamily of the aquaporin family, and are permeable to both water and glycerol (2). Apart from the transport of small molecules, the AQPs are involved in a variety of biological processes, including tissue swelling (3), glucolipid metabolism (4), neural signal transduction (5) and cell migration (6). AQP9 is a unique AQP channel in hepatocytes, while AQP3 and AQP7 act as glycerol channels in adipocytes (7). The three AQP subtypes serve key roles in glucolipid metabolism $(8,9)$.

Accumulating evidence indicates a close association between the expression of AQPs and carcinogenesis (10). For instance, AQP3 is expressed in human esophageal and oral squamous cell carcinoma, and contributes to tumor cell growth (11). Tan et al (12) reported that the expression of AQP9 is significantly higher in human astrocytic tumors compared with that in normal brain tissues, and is positively correlated with pathological grade. Hepatocellular carcinoma (HCC) is one of the most common gastrointestinal malignancies worldwide, with a particularly high incidence in Asian countries (13). It has been previously documented that combined overexpression of AQP3 and AQP5 is an independent poor prognostic factor for HCC (14). Decreased expression levels of AQP8 and AQP9 has been revealed to confer apoptosis resistance in a rat HCC line (15). These previous studies suggest that AQPs are involved in the development and progression of HCC. However, the expression and clinical significance of glycolipid metabolism-associated AQPs (AQP3, 7 and 9) in HCC remains to be fully elucidated.

Therefore, the present study assessed the mRNA and protein expression levels of AQP3, 7 and 9 in human HCCs and adjacent non-tumorous liver (NTL) tissues, and explored the association of AQP proteins with the clinicopathological features of HCC.

\section{Materials and methods}

Tissue specimens. The present study enrolled a total of $68 \mathrm{HCC}$ patients who underwent hepatectomy at the affiliated hospitals 
of Chongqing Medical University (Chongqing, China) between October 2009 and May 2013. All patients were pathologically diagnosed with HCC. Fresh tumor samples, coupled with adjacent NTL tissues, were collected from each patient. Some of the excised tissues were placed immediately in liquid nitrogen and stored at $-80^{\circ} \mathrm{C}$ until gene expression analysis. The other tissue samples were fixed, paraffin-embedded and sectioned (4 $\mu \mathrm{m}$; OML-QPA/QPB; Hubei OML Medical Science \& Technology Co., Ltd., Xiaogan, China) for immunohistochemistry. Written informed consent was obtained from each patient and the study protocol was approved by the Ethics Committee of Chongqing Medical University.

Reverse transcription-quantitative polymerase chain reaction $(R T-q P C R)$. The total RNA was extracted from the tissue samples using the RNAiso Plus reagent (Takara Bio, Inc., Tokyo, Japan). cDNA was synthesized from the total RNA using the PrimeScript RT reagent Kit (Takara Bio, Inc.). RT-qPCR was performed on a CFX96 Real-Time System PCR detecting system (Bio-Rad Laboratories, Hercules, CA, USA) using the SYBR Premix Ex Taq II kit (Takara Bio, Inc.). The primers used are as follows: AQP3, sense: 5'-CCTCTG GACACTTGGATATGAT-3' and antisense: 5'-GGGACG GGGTTGTTGTAG-3'; AQP7, sense: 5'-CCGCATCTTCAC CTTCATTG-3' and antisense: 5'-CACCCACCACCAGTT CTC-3'; AQP9, sense: 5'-ATCCACCAGAAGTTGTTT-3' and antisense 5'-AGCAATGACAATAATCAGGAGGC-3'. For the control, glyceraldehyde-3-phosphate dehydrogenase (GAPDH) was amplified in a parallel reaction, with the following primers: GAPDH, sense: 5'-GGTGGTCTCCTCTGACTTCAACA-3' and antisense: 5'-GTTGCTGTAGCCAAATTCGTTGT-3'. The cycling conditions were as follows: Initial denaturation at $95^{\circ} \mathrm{C}$ for $3 \mathrm{~min}$, followed by 40 cycles of denaturation at $95^{\circ} \mathrm{C}$ for $10 \mathrm{sec}$ and annealing at $60^{\circ} \mathrm{C}$ for $30 \mathrm{sec}$. The data were analyzed using the $2^{-\Delta \Delta C q}$ method (16). The relative mRNA levels were calculated following normalization against GAPDH mRNA levels.

Western blot analysis. Tissue samples were homogenized in radioimmunoprecipitation lysis buffer (Beyotime Institute of Biotechnology, Shanghai, China) containing $1 \%$ sodium dodecyl sulfate (SDS) and 1\% phenylmethylsulfonyl fluoride (Sigma-Aldrich, St. Louis, MO, USA), a potent protease inhibitor. The protein concentrations were measured using the bicinchoninic acid Protein Assay kit (Pierce, Rockford, IL, USA). Equal quantities of the total protein $(\sim 100 \mu \mathrm{g})$ were separated by $12 \%$ SDS-polyacrylamide gel electrophoresis and were transferred onto a polyvinylidene fluoride membrane. The membrane was blocked at room temperature for $1 \mathrm{~h}$ with $5 \%$ fat-free milk, and incubated overnight with primary antibodies at $4^{\circ} \mathrm{C}$. The following primary antibodies were used: Mouse monoclonal anti-GAPDH (1:500; cat. no. TA-08; ZSGB-BIO Co., Ltd., Beijing, China), rabbit polyclonal anti-AQP3 (1:500; cat. no. LS-B8185; LifeSpan Biosciences, Inc., Seattle, WA, USA), rabbit polyclonal anti-AQP7 (1:300; cat. no. ab85907; Abcam, Cambridge, MA, USA) and rabbit polyclonal anti-AQP9 (1:500; cat. no. ab85910; Abcam). The membranes were washed with Tris-buffered saline and Tween 20 buffer (ZSGB-BIO Co., Ltd.) and incubated for $1 \mathrm{~h}$ at room temperature with goat anti-rabbit (cat. no. ZB-2301) or anti-mouse immunoglobulin G (ZB-2305)(1:3,000; ZSGB-BIO Co., Ltd.). The proteins were visualized using an enhanced chemiluminescence detection kit (ECL plus; Beyotime Institute of Biotechnology). Relative band intensities (AQP/GAPDH protein ratios) were determined by densitometry using the Quality One software (version 4.62; Bio-Rad Laboratories).

Immunohistochemistry. Tissue specimens were embedded in paraffin and cut into $4 \mu \mathrm{m}$ sections. The tissue sections were dewaxed in xylene, rehydrated, and heated in citrate buffer (ZSGB-BIO Co., Ltd.) for $20 \mathrm{~min}$ at $100^{\circ} \mathrm{C}$ to retrieve antigen. Following the elimination of endogenous peroxidase, the tissue sections were blocked with $3 \%$ hydrogen peroxidase diluted with methyl alcohol for 20 min and washed with phosphate-buffered saline (PBS), and incubated overnight at $4^{\circ} \mathrm{C}$ with anti-AQP3 (1:100), anti-AQP7 (1:200) or anti-AQP9 (1:200) primary antibodies. Negative controls were included by omitting the primary antibody. The membranes were then washed with PBS for $10 \mathrm{~min}$, and the secondary antibody reaction was performed using the Polink-2 plus Polymer Horseradish Peroxidase Detection system (GBI Labs, Mukilteo, WA, USA), according to the manufacturer's protocol. The tissue sections were developed with 3,3-diaminobenzidine (ZSGB-BIO Co., Ltd.) and were counterstained with hematoxylin. The stained sections were independently assessed by two pathologists in a blinded manner. The median percentage of immunostained tumor cells $(10 \%)$ was used as a cutoff. High expression of AQPs was defined as nuclear staining of $\geq 10 \%$ of the tumor cells and low expression of AQPs was defined as nuclear staining of $<10 \%$ of the tumor cells or no nuclear staining.

Statistical analysis. The data are presented as the mean \pm standard deviation. All statistical calculations were performed using SPSS version 18.0 (IBM SPSS, Chicago, IL, USA). Continuous data were compared using the paired Student's t-test. The correlation between the expression levels of AQP3, 7 and 9 and the association between AQP expression and the clinicopathological features of $\mathrm{HCC}$ were analyzed using the $\chi^{2}$ test. $\mathrm{P}<0.05$ was considered to indicate a statistically significant difference.

\section{Results}

mRNA expression levels of AQPs in HCC. RT-qPCR analysis revealed that compared with NTL tissue, HCC tissues exhibited a significant $(\mathrm{P}<0.05)$ increase in the AQP3 mRNA level and a concomitant reduction in the mRNA expression levels of AQP7 and AQP9 (Fig. 1).

Protein expression levels of $A Q P$ s in $H C C$. Western blot analysis confirmed an upregulation of AQP3 and downregulation of AQP7 and AQP9 in HCC compared with in the NTL tissue (Fig. 2). Immunohistochemistry was performed to determine the location and distribution of AQPs in HCC. AQP9 was localized on the plasma membrane and in the cytoplasm of hepatocytes, while AQP3 and AQP7 showed predominantly cytoplasmic and nuclear distribution (Fig. 3). The majority of HCC tissues exhibited a significant decrease 

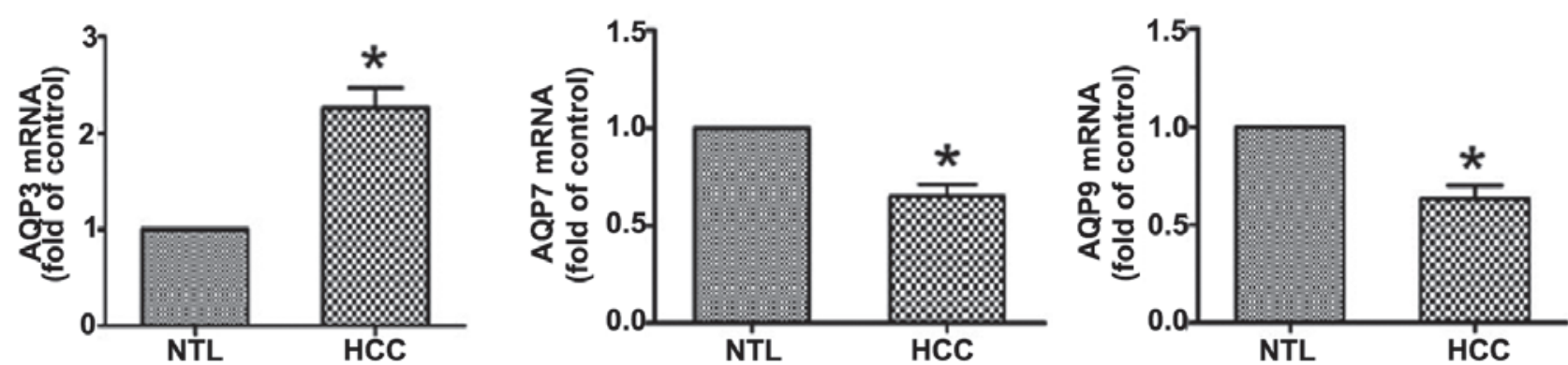

Figure 1. Reverse transcription-quantitative polymerase chain reaction analysis of the mRNA expression levels of AQP3, AQP7, and AQP9 in 68 pairs of HCC and NTL tissues. The data are presented as the mean \pm standard deviation ( $\mathrm{P}<0.05)$. AQP, aquaglyceroporins; HCC, hepatocellular carcinoma; NTL, non-tumorous liver.
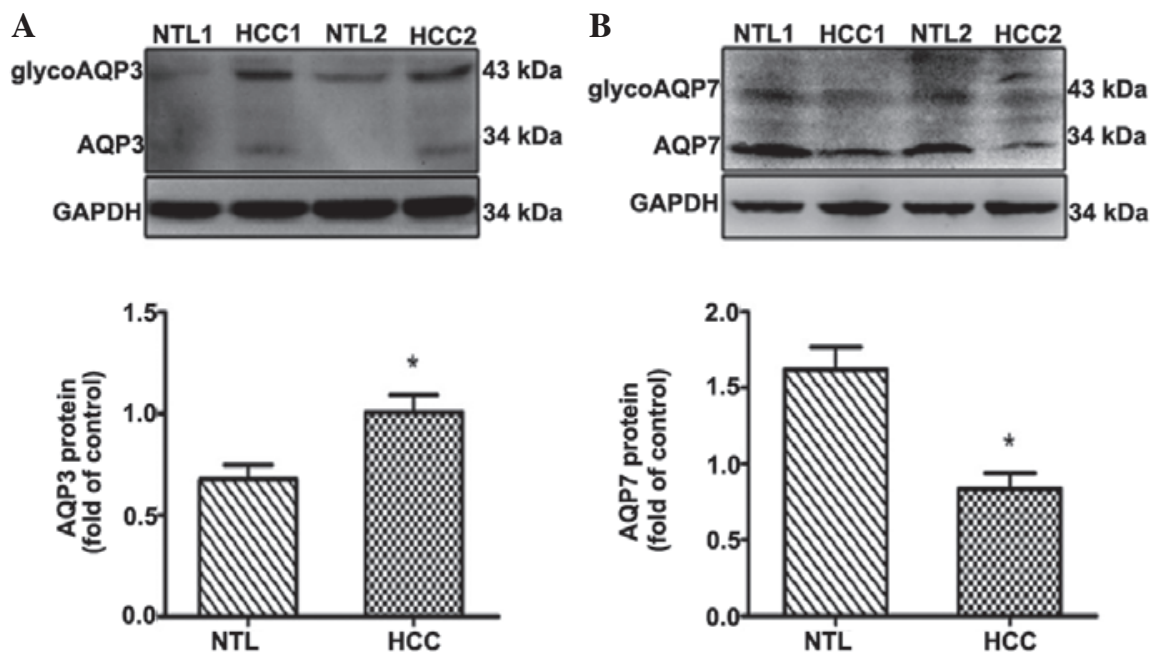
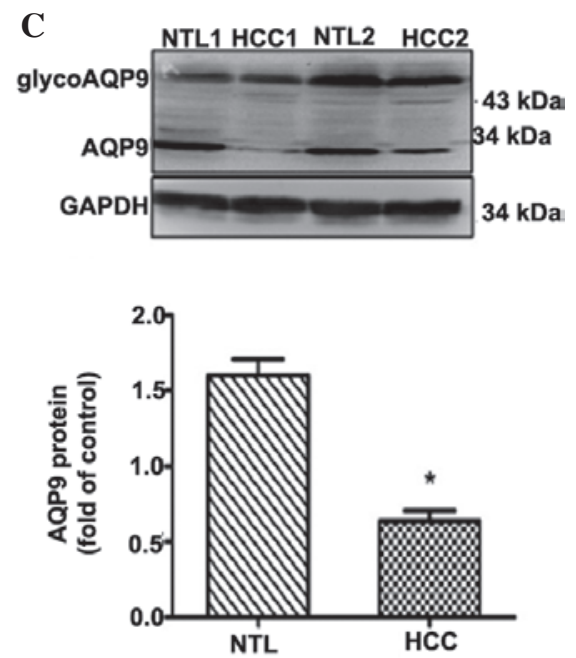

Figure 2. Western blot analysis of the protein expression levels of (A) AQP3, (B) AQP7 and (C) AQP9 in 68 pairs of HCC and adjacent NTL tissues. Representative western blotting is shown in the upper panels. Bar graphs (lower panels) represent densitometric analysis of the blots from three independent experiments. The data are presented as the mean \pm standard deviation $(" \mathrm{P}<0.05)$. The antibodies detect glycosylated and non-glycosylated forms of AQP. $\mathrm{AQP}$, aquaglyceroporins; HCC, hepatocellular carcinoma; NTL, non-tumorous liver.
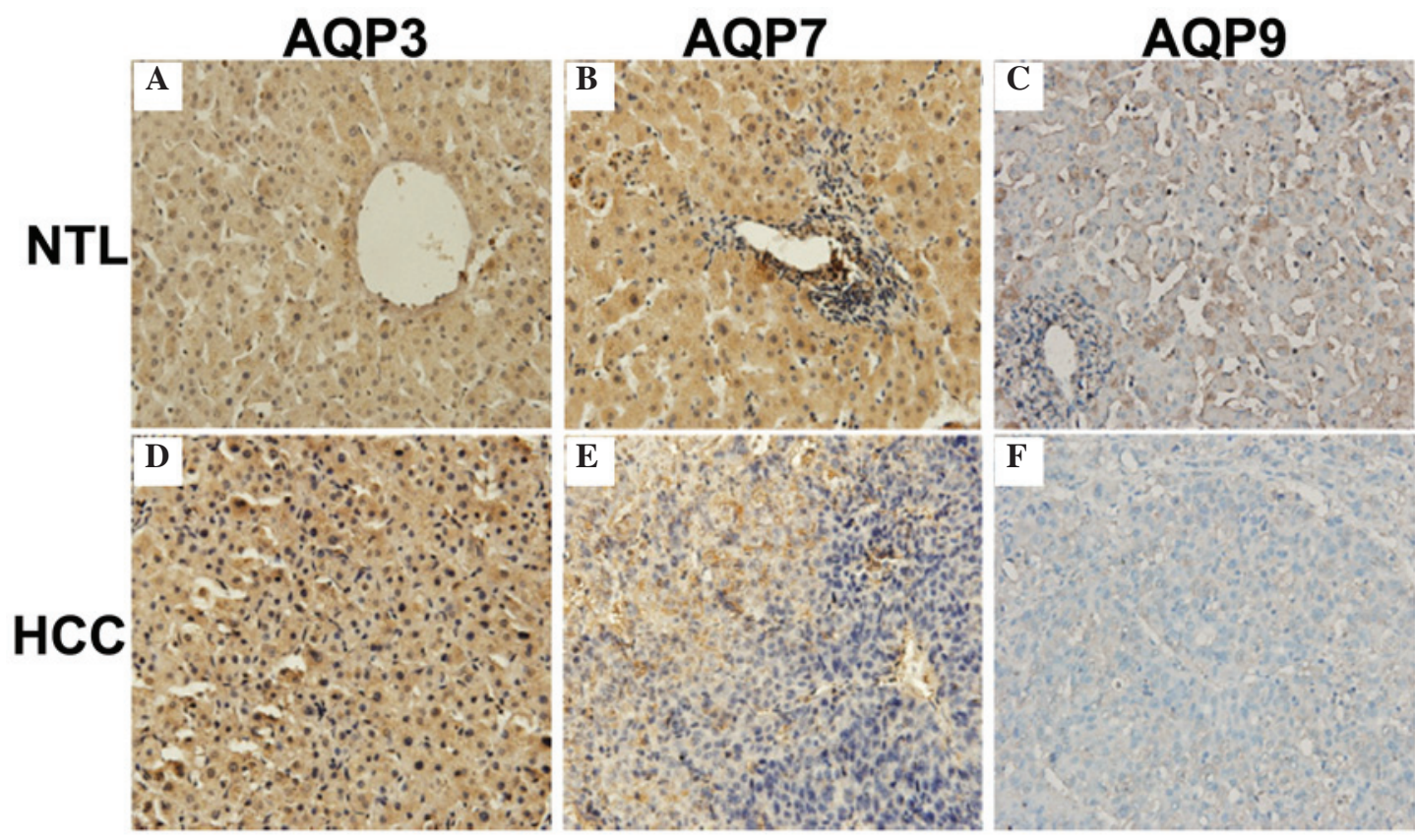

Figure 3. Assessment of histological location and distribution of AQPs by immunohistochemistry. Representative images of NTL tissues stained with (A) anti-AQP3, (B) anti-AQP7 and (C) anti-AQP9 antibodies, and HCC tissues stained with (D) anti-AQP3, (E) anti-AQP7 and (F) anti-AQP9 antibodies. Magnification, x400. AQP, aquaglyceroporins; HCC, hepatocellular carcinoma; NTL, non-tumorous liver. 
Table I. Altered mRNA expression levels of AQP3, 7 and 9 in human HCC tissues and pair-matched NTL tissues.

\begin{tabular}{lccc}
\hline Group & AQP3 & AQP7 & AQP9 \\
\hline NTL (n=68) & $1.000 \pm 0.000$ & $1.000 \pm 0.000$ & $1.000 \pm 0.000$ \\
HCC (n=68) & $2.259 \pm 0.210$ & $0.652 \pm 0.487$ & $0.636 \pm 0.069$ \\
t-test & 3.025 & 4.928 & 6.596 \\
P-value & $<0.0001$ & $<0.0001$ & $<0.0001$ \\
R-value & 0.120 & 0.266 & 0.394 \\
$95 \%$ CI & $(-0.546)-(-0.112)$ & $0.467-1.104$ & $0.672-1.256$
\end{tabular}

Paired sample t-test (NTL vs HCC). NTL, non-tumorous liver tissue; HCC, hepatocellular carcinoma; CI, confidence intervals.

Table II. Correlation analysis of the protein expression levels of AQP3, AQP7 and AQP9 in HCC tissues (n=68).

\begin{tabular}{|c|c|c|c|c|c|}
\hline & \multicolumn{2}{|r|}{ AQP3 } & \multirow[b]{2}{*}{$\chi^{2}$} & \multirow[b]{2}{*}{$\mathrm{r}$} & \multirow[b]{2}{*}{ P-value } \\
\hline & High $(n=46)$ & Low $(n=22)$ & & & \\
\hline \multicolumn{6}{|l|}{ AQP7 } \\
\hline $\operatorname{High}(n=12)$ & 5 & 7 & 4.494 & -0.479 & $<0.05$ \\
\hline Low $(n=56)$ & 41 & 15 & & & \\
\hline \multicolumn{6}{|l|}{ AQP9 } \\
\hline High $(n=10)$ & 4 & 6 & 4.095 & -0.448 & $<0.05$ \\
\hline Low $(n=58)$ & 42 & 16 & & & \\
\hline
\end{tabular}

in the expression levels of AQP7 and AQP9, compared with adjacent NTL tissues $(\mathrm{P}<0.0001$ for each comparison; Table I). By contrast, an increased expression of AQP3 was observed in HCC compared with the NTL tissues $(\mathrm{P}=0.0035$; Table I).

Correlation between the expression levels of AQP3, 7 and 9. To determine whether a correlation exists between the protein expression levels of AQP3, 7 and 9 in HCC tissues, a $\chi^{2}$ test was performed. As shown in Table II, high expression of AQP3 was significantly $(\mathrm{P}<0.05)$ associated with low expression of AQP7 $(r=0.479 ; \mathrm{P}<0.05)$ and AQP9 $(r=0.448 ; \mathrm{P}<0.05)$ in HCC tissues. However, no significant correlation was observed between AQP7 and AQP9 (data not shown)

Correlation between AQP proteins and the clinicopathological parameters of $H C C$. Clinicopathological features and the expression of AQP proteins in the $68 \mathrm{HCC}$ patients were summarized in Table III. High expression of AQP3 correlated with tumor grade $\left(\chi^{2}=5.740 ; \mathrm{P}=0.017\right)$, tumor stage $\left(\chi^{2}=6.680\right.$; $\mathrm{P}=0.010)$ and lymphatic metastasis $\left(\chi^{2}=4.636 ; \mathrm{P}=0.031\right)$. Low expression of AQP7 was correlated with tumor grade $\left(\chi^{2}=4.091\right.$; $\mathrm{P}=0.043$ ). However, the expression of AQP9 protein revealed no association with any of the clinicopathological factors studied.

\section{Discussion}

As water transporters, AQPs are expressed in a variety of tissues and cells (1,17). Accumulating evidence indicates that AQPs are frequently dysregulated in cancer and serve critical roles in tumor development and progression (10). The expression of AQP3 has been shown to be upregulated in colorectal carcinoma (18), gastric cancer (19), cervical cancer (20) and HCC (14). Guo et al (14) reported that elevated expression of AQP3 and AQP5, as determined by immunohistochemistry, is significantly associated with tumor progression and prognosis in patients with $\mathrm{HCC}$. The present data confirmed the upregulation of AQP3 in HCC compared with that in adjacent NTL tissue. Additionally, it was revealed that the mRNA and protein expression levels of AQP3 were consistently increased in HCC, suggesting that the upregulation of AQP3 likely occurs at the transcriptional level. It has been documented that the expression of AQP9 is significantly decreased in HCC tissues compared with NTL tissues (21). In agreement with this previous study, the present results demonstrated that both the mRNA and protein expression levels of AQP9 were reduced in HCC compared with the adjacent NTL tissue. The downregulation of AQP9 has been shown to induce apoptosis resistance in HCC cells (21). Notably, AQP7 has been identified in human urothelial carcinoma (22). The present study provided the first evidence, to the best of our knowledge, that AQP7 was downregulated in HCC compared with adjacent NTL tissue. This downregulation may be due to transcriptional inhibition, since the mRNA and protein expression levels of AQP7 were consistently decreased. Taken together, the present data demonstrated that HCCs exhibited coordinated expression of AQPs. However, the exact mechanisms for their dysregulation require further clarified.

By immunohistochemistry, AQPs exhibited different localization patterns in HCC. It was found that AQP9 was localized on the plasma membrane and in the cytoplasm of hepatocytes, while AQP3 and AQP7 exhibited cytoplasmic 


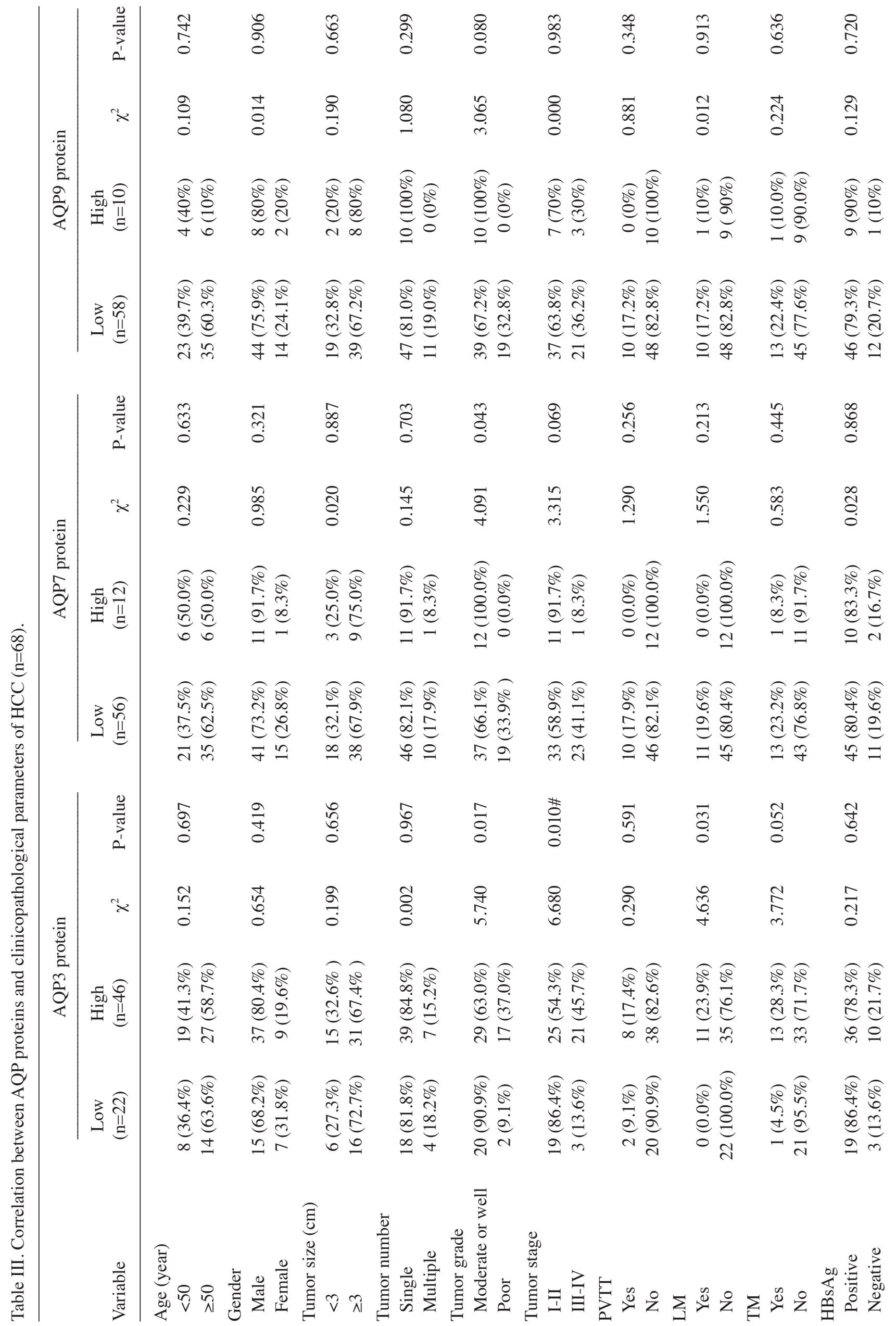


and nuclear distribution. Nihei et al (23) reported that AQP9 is normally localized on the surface of rat hepatocytes and Leydig cells. Similarly, Elkjaer et al (24) demonstrated an immunolocalization of AQP9 on the plasma membrane of liver hepatocytes. The data from knockout mice support the essential role for AQP9 in glycerol transport (25). Previous studies, combined with the present findings, suggested that AQP9 may facilitate the transport of water, glycerol and other small molecules in HCC cells. The cytoplasmic and nuclear expression pattern of AQP3 and AQP7 suggested that the two proteins may be involved in the regulation of gene expression. Indeed, Xie et al (26) demonstrated that AQP3 has a protective activity against ultraviolet A-induced human skin fibroblast apoptosis via the upregulation of B-cell lymphoma-2. AQP3 depletion has been shown to induce the expression of p21 and FAS in cancer cells (27). Forced expression of AQP7 leads to improved insulin resistance by increasing the phosphorylation of protein kinase B (28). Taken together, different expression and localization patterns of AQPs in HCC indicate their distinct roles in tumor progression.

The present data demonstrated that high expression of AQP3 correlated with tumor grade, tumor stage and lymphatic metastasis in HCC, suggesting its favorable role in HCC progression. The tumor-promoting effects of AQP 3 have also been previously described in several other cancer types. For instance, Chen et al (29) reported that AQP3 facilitates the epithelial-mesenchymal transition in gastric cancer. Li et al (18) revealed that AQP3 overexpression promotes colorectal carcinoma cell migration and is significantly associated with tumor metastasis. Different from AQP3, low expression of AQP7 was found to be significantly correlated with tumor grade in HCC patients, implying that AQP7 may exert suppressive effects on HCC. Although the protein expression of AQP9 exhibited no significant association with HCC clinicopathological features, low expression of AQP9 was significantly associated with high expression of AQP3 in patients with HCC. It has been documented that AQP9 and AQP7 are implicated in the uptake of certain chemotherapeutic agents into cancer cells $(30,31)$. Decreased expression of AQP9 is associated with increased resistance to apoptosis in HCC cells (15). Therefore, the dysregulation of $\mathrm{AQP} 3,7$ and 9 may cooperatively contribute to the pathogenesis of HCC. However, their biological functions in HCC requires further elucidation.

In conclusion, the present data demonstrated that $\mathrm{AQP} 3$ is upregulated, and that $\mathrm{AQP7}$ and $\mathrm{AQP} 9$ are downregulated in HCC. It was also revealed that the three investigated AQPs exhibit different intracellular localizations in HCC hepatocytes. High expression of AQP3 was significantly associated with tumor grade, tumor stage and lymphatic metastasis in patients with HCC, while low expression of AQP7 was significantly correlated with tumor grade. The present results suggest a complex role for AQPs in the development and progression of HCC. Additional direct studies are required to determine the biological functions of AQPs in this malignancy.

\section{References}

1. Ishibashi K, Hara S and Kondo S: Aquaporin water channels in mammals. Clin Exp Nephrol 13: 107-117, 2009. 
2. Mukhopadhyay $\mathrm{R}$, Bhattacharjee $\mathrm{H}$ and Rosen $\mathrm{BP}$ Aquaglyceroporins: Generalized metalloid channels. Biochim Biophys Acta 1840: 1583-1591, 2014.

3. Papadopoulos MC and Verkman AS: Aquaporin-4 gene disruption in mice reduces brain swelling and mortality in pneumococca meningitis. J Biol Chem 280: 13906-13912, 2005.

4. Calamita G, Gena P, Ferri D, Rosito A, Rojek A, Nielsen S, Marinelli RA, Frühbeck G and Svelto M: Biophysical assessment of aquaporin-9 as principal facilitative pathway in mouse liver import of glucogenetic glycerol. Biol Cell 104: 342-351, 2012.

5. Amiry-Moghaddam M, Williamson A, Palomba M, Eid T, de Lanerolle NC, Nagelhus EA, Adams ME, Froehner SC, Agre P and Ottersen OP: Delayed K+ clearance associated with aquaporin- 4 mislocalization: Phenotypic defects in brains of alpha-syntrophin-null mice. Proc Natl Acad Sci USA 100 13615-13620, 2003.

6. Hara-Chikuma M and Verkman AS: Aquaporin-1 facilitates epithelial cell migration in kidney proximal tubule. J Am Soc Nephrol 17: 39-45, 2006.

7. Rodríguez A, Catalán V, Gómez-Ambrosi J, García-Navarro S, Rotellar F, Valentí V, Silva C, Gil MJ, Salvador J, Burrell MA, et al: Insulin- and leptin-mediated control of aquaglyceroporins in human adipocytes and hepatocytes is mediated via the PI3K/Akt/mTOR signaling cascade. J Clin Endocrinol Metab 96: E586-E597, 2011.

8. Maeda N: Implications of aquaglyceroporins 7 and 9 in glycerol metabolism and metabolic syndrome. Mol Aspects Med 33: 665-675, 2012.

9. Rodríguez A, Catalán V, Gómez-Ambrosi J and Frühbeck G: Aquaglyceroporins serve as metabolic gateways in adiposity and insulin resistance control. Cell Cycle 10: 1548-1556, 2011.

10. Ribatti D, Ranieri G, Annese T and Nico B: Aquaporins in cancer Biochim Biophys Acta 1840: 1550-1553, 2014.

11. Kusayama M, Wada K, Nagata M, Ishimoto S, Takahashi H Yoneda M, Nakajima A, Okura M, Kogo M and Kamisaki Y: Critical role of aquaporin 3 on growth of human esophageal and oral squamous cell carcinoma. Cancer Sci 102: 1128-1136, 2011.

12. Tan G, Sun SQ and Yuan DL: Expression of the water channel protein aquaporin-9 in human astrocytic tumours: Correlation with pathological grade. J Int Med Res 36: 777-782, 2008.

13. Venook AP, Papandreou C, Furuse J and de Guevara LL: The incidence and epidemiology of hepatocellular carcinoma: A global and regional perspective. Oncologist 15 (Suppl 4): S5-S13, 2010.

14. Guo X, Sun T, Yang M, Li Z, Li Z and Gao Y: Prognostic value of combined aquaporin 3 and aquaporin 5 overexpression in hepatocellular carcinoma. Biomed Res Int 2013: 206525, 2013.

15. Jablonski EM, Mattocks MA, Sokolov E, Koniaris LG Hughes FM Jr, Fausto N, Pierce RH and McKillop IH: Decreased aquaporin expression leads to increased resistance to apoptosis in hepatocellular carcinoma. Cancer Lett 250: 36-46, 2007.

16. Livak KJ and Schmittgen TD: Analysis of relative gene expression data using real-time quantitative PCR and the 2 (-Delta Delta C (T)) Method. Methods 25: 402-408, 2001.
17. Verkman AS, Anderson MO and Papadopoulos MC: Aquaporins: Important but elusive drug targets. Nat Rev Drug Discov 13: 259-277, 2014.

18. Li A, Lu D, Zhang Y, Li J, Fang Y, Li F and Sun J: Critical role of aquaporin-3 in epidermal growth factor-induced migration of colorectal carcinoma cells and its clinical significance. Oncol Rep 29: 535-540, 2013.

19. Chen J, Wang T, Zhou YC, Gao F, Zhang ZH, Xu H, Wang SL and Shen LZ: Aquaporin 3 promotes epithelial-mesenchymal transition in gastric cancer. J Exp Clin Cancer Res 33: 38, 2014

20. Chen R, Shi Y, Amiduo R, Tuokan T and Suzuk L: Expression and prognostic value of aquaporin 1, 3 in cervical carcinoma in women of Uygur ethnicity from Xinjiang, China. PLoS One 9: e98576, 2014

21. Jablonski EM, Mattocks MA, Sokolov E, Koniaris LG, Hughes FM Jr, Fausto N, Pierce RH and McKillop IH: Decreased aquaporin expression leads to increased resistance to apoptosis in hepatocellular carcinoma. Cancer Lett 250: 36-46, 2007.

22. Rubenwolf PC, Otto W, Denzinger S, Hofstädter F, Wieland W and Georgopoulos NT: Expression of aquaporin water channels in human urothelial carcinoma: Correlation of AQP3 expression with tumour grade and stage. World J Urol 32: 991-997, 2014.

23. Nihei K, Koyama Y, Tani T, Yaoita E, Ohshiro K, Adhikary LP, Kurosaki I, Shirai Y, Hatakeyama K and Yamamoto T: Immunolocalization of aquaporin-9 in rat hepatocytes and Leydig cells. Arch Histol Cytol 64: 81-88, 2001.

24. Elkjaer M, Vajda Z, Nejsum LN, Kwon T, Jensen UB, Amiry-Moghaddam M, Frøkiaer $J$ and Nielsen S: Immunolocalization of AQP9 in liver, epididymis, testis, spleen, and brain. Biochem Biophys Res Commun 276: 1118-1128, 2000.

25. Liu Y, Promeneur D, Rojek A, Kumar N, Frøkiaer J, Nielsen S, King LS, Agre P and Carbrey JM: Aquaporin 9 is the major pathway for glycerol uptake by mouse erythrocytes, with implications for malarial virulence. Proc Natl Acad Sci USA 104: 12560-12564, 2007.

26. Xie H, Liu F, Liu L, Dan J, Luo Y, Yi Y, Chen X and Li J: Protective role of AQP3 in UVA-induced NHSFs apoptosis via Bcl2 up-regulation. Arch Dermatol Res 305: 397-406, 2013.

27. Trigueros-Motos L, Pérez-Torras S, Casado FJ, Molina-Arcas M and Pastor-Anglada M: Aquaporin 3 (AQP3) participates in the cytotoxic response to nucleoside-derived drugs. BMC Cancer 12: 434, 2012

28. Shen FX, Gu X, Pan W, Li WP, Li W, Ye J, Yang LJ, Gu XJ and Ni LS: Over-expression of AQP7 contributes to improve insulin resistance in adipocytes. Exp Cell Res 318: 2377-2384, 2012.

29. Chen J, Wang T, Zhou YC, Gao F, Zhang ZH, Xu H, Wang SL and Shen LZ: Aquaporin 3 promotes epithelial-mesenchymal transition in gastric cancer. J Exp Clin Cancer Res 33: 38, 2014.

30. Liu Z, Shen J, Carbrey JM, Mukhopadhyay R, Agre P and Rosen BP: Arsenite transport by mammalian aquaglyceroporins AQP7 and AQP9. Proc Natl Acad Sci USA 99: 6053-6058, 2002.

31. Bhattacharjee H, Carbrey J, Rosen BP and Mukhopadhyay R: Drug uptake and pharmacological modulation of drug sensitivity in leukemia by AQP9. Biochem Biophys Res Commun 322: 836-841, 2004. 